\title{
Limited flexibility and unusual longevity shape forager allocation in the Florida harvester ant (Pogonomyrmex badius)
}

\author{
Christina L. Kwapich ${ }^{1}$ - Walter R. Tschinkel ${ }^{2}$
}

Published online: 14 March 2016

(C) Springer-Verlag Berlin Heidelberg 2016

\begin{abstract}
The benefits of behavioral flexibility in social insect societies are well known, but the advantages of limited flexibility have seldom been considered. Florida harvester ant colonies maintain a stable forager population size for much of their active season, and despite seasonal variation in chronological age, foragers die within 27 days of initiating foraging. To determine how colonies balance forager mortality and forager replacement, we tested the relative influences of intrinsic and extrinsic factors on forager membership, retention, and longevity. Potential and realized forager longevity differed significantly. Residual lifespan increased by $57 \%$ when colonies were penned for 20 days, and up to 8 -fold when foragers were retained in the laboratory. Increased forager longevity inhibited the movement of new workers into the forager population. In contrast, increased mortality and starvation did not stimulate the addition of new foragers and forager population size declined when mortality exceeded $4 \%$ per day. Experimental increases in forager number, body
\end{abstract}

fat, and the ratio of larvae to foragers did not induce behavioral reversion in existing foragers. These results suggest an unidirectional allocation strategy, with foragers that are less disposable and less behaviorally flexible than the wellstudied honey bee. In P. badius, forager membership is maintained not by young ants detecting increased demand, but by workers developing at rates that allow forager replacement and prevent excessive worker depletion. In the absence of a lifespan matched to predictable risks, opportunistic increases in forager survival may promote colony growth by inhibiting the scheduled and irreversible transitions of younger workers.

This article was originally intended as an invited contribution to the special issue on the Integrative Analysis of Division of Labor but was inadvertently published prematurely in Behavioral Ecology and Sociobiology, Volume 70, Issue 2, pp 221-235, DOI 10.1007/s00265-015-2039-1.
Christina L. Kwapich

ckwapich@asu.edu School of Life Sciences, Arizona State University, Tempe, AZ, USA

2 Department of Biological Science, Florida State University, Tallahassee, FL, USA 